\title{
Une base symétrique de l'algèbre des coinvariants quasi-symétriques
}

\author{
Frédéric Chapoton \\ Institut Camille Jordan \\ Université Claude Bernard Lyon 1 \\ Bâtiment Braconnier \\ 21 Avenue Claude Bernard \\ F-69622 VILLEURBANNE Cedex, FRANCE \\ chapoton@math. univ-lyon1.fr
}

Submitted: Jun 10, 2005; Accepted: Sep 9, 2005; Published: Sep 19, 2005

Mathematics Subject Classifications: 05E05

\begin{abstract}
Résumé
On décrit une nouvelle base de l'algèbre des coinvariants quasi-symétriques, qui est stable par l'involution naturelle et indexée par les triangulations d'un polygone régulier.
\end{abstract}

\begin{abstract}
We describe a new basis of the ring of quasi-symmetric coinvariants, which is stable by the natural reversal of the set of variables. The indexing set is the set of triangulations of a regular polygon, instead of the set of Dyck paths used for the known basis.
\end{abstract}

\section{Introduction}

L'algèbre des coinvariants est un objet classique associé à chaque groupe de Coxeter fini $W[6]$. Cette algèbre est définie comme le quotient de l'algèbre des polynômes sur l'espace vectoriel sur lequel $W$ agit par réflexions, par l'idéal homogène engendré par les polynômes invariants homogènes non constants. Le quotient est une algèbre graduée de dimension finie donnée par l'ordre de $W$.

Dans le cas du groupe symétrique sur $n+1$ lettres, on peut expliciter cette construction comme le quotient de l'algèbre des polynômes en $\mathrm{x}_{1}, \ldots, \mathrm{x}_{n+1}$ par l'idéal homogène engendré par les fonctions symétriques élémentaires. Plus récemment, une notion plus faible que celle de polynôme symétrique est apparue [5]. Un polynôme est dit quasi-symétrique 
si, pour toute suite d'exposants $\left(m_{1}, \ldots, m_{k}\right)$ fixée, tous les monômes $x_{i_{1}}^{m_{1}} \ldots \mathrm{x}_{i_{k}}^{m_{k}}$ pour une suite croissante d'indices $i_{1}<i_{2}<\cdots<i_{k}$ ont le même coefficient. En particulier, les polynômes symétriques sont aussi quasi-symétriques.

Dans [2, 1], la notion d'algèbre coinvariante quasi-symétrique a été introduite et étudiée. Elle est définie comme le quotient de l'algèbre des polynômes en $x_{1}, \ldots, x_{n+1}$ par l'idéal homogène engendré par les polynômes quasi-symétriques homogènes non constants. C'est une algèbre graduée. Il est démontré dans [1] que cette algèbre est de dimension finie, donnée par le nombre de Catalan $c_{n+1}$. La preuve est la construction explicite d'un ensemble de monômes indexés par les chemins de Dyck de longueur $2 n+2$, dont les images dans le quotient forment une base de l'algèbre des coinvariants quasi-symétriques.

Dans le cas des coinvariants usuels, le groupe de Coxeter $W$ agit par automorphismes sur le quotient et on obtient une décomposition intéressante du module régulier. Dans le cas des coinvariants quasi-symétriques, le seul automorphisme de la situation est le renversement qui envoie $\mathrm{x}_{i}$ sur $\mathrm{x}_{n+2-i}$. Cette involution préserve l'idéal des fonctions quasisymétriques sans terme constant et passe donc au quotient.

La motivation initiale de cet article est le fait que l'action de cette involution semble difficile à décrire dans la base des monômes associés aux chemins de Dyck. On construit donc une nouvelle base, dans laquelle l'involution agit par permutation. Cette base est formée de polynômes dont le terme dominant pour l'ordre naturel sur les variables $\mathrm{x}_{1}, \ldots, \mathrm{x}_{n+1}$ redonne les monômes associés aux chemins de Dyck.

Il apparaît que l'ensemble naturel d'indexation de cette nouvelle base est non pas l'ensemble des chemins de Dyck, mais celui des triangulations d'un polygone régulier. Cet ensemble joue un rôle primordial dans la théorie des algèbres à grappes de Fomin et Zelevinsky [4]. Cet article donne donc un premier rapprochement entre les algèbres à grappes et les fonctions quasi-symétriques. Il se trouve que la construction de la base indexée par les triangulations passe par le choix d'une triangulation de base en forme d'éventail. Dans le cadre de la théorie des algèbres à grappes, ce choix correspond au carquois équi-orienté de type $A_{n}$, voir par exemple [3].

L'article est organisé comme suit. On commence par définir une bijection ad hoc entre triangulations et chemins de Dyck. Ensuite on montre que, par cette bijection, le monôme dominant du polynôme associé à une triangulation est le monôme associé au chemin de Dyck correspondant, ce qui entraîne immédiatement le résultat principal.

\section{Bijection}

Soit $n$ un entier positif ou nul. On définit dans cette section une bijection entre

1. les triangulations d'un polygone régulier à $n+3$ cotés,

2. les chemins de Dyck de longueur $2 n+2$.

Il est bien connu que ces deux ensembles ont pour cardinal le nombre de Catalan

$$
c_{n+1}=\frac{1}{n+2}\left(\begin{array}{c}
2 n+2 \\
n+1
\end{array}\right)
$$


Par définition, un chemin de Dyck est une suite de pas verticaux ("montées") et horizontaux ("descentes") qui reste au dessus de la diagonale, voir la partie droite de la figure 1 .

La bijection est illustrée par un exemple dans la figure 1.

Avant toute chose, on fixe une triangulation de base en forme d'éventail, c'est-àdire formée par toutes les diagonales contenant un sommet choisi, noté \#. On dessine cette triangulation avec le sommet commun à toutes les diagonales placé en bas. Les diagonales de cette triangulation de base seront dites "négatives" et numérotées de 1 à $n$ de gauche à droite. Les diagonales qui n'interviennent pas dans la triangulation de base sont dites "positives". On numérote aussi de 1 à $n$ les sommets aux extrémités des diagonales négatives.

On associe alors un chemin de Dyck $D(T)$ à chaque triangulation $T$, par récurrence sur $n$. Pour $n=0$, à la seule triangulation du polygone à trois cotés est associée le seul chemin de Dyck de longueur 2.

Si $n$ est non nul, on regarde le sommet $*$ du polygone placé à droite du sommet \# dans le sens trigonométrique. On distingue deux cas.

Si le sommet $*$ participe à un seul triangle de la triangulation $T$ i.e. n'est contenu dans aucune diagonale de $T$, on lui associe le chemin de Dyck obtenu en encadrant par une montée et une descente le chemin de Dyck $D\left(T^{\prime}\right)$ associé à la triangulation $T^{\prime}$ du polygone à $n+2$ cotés qui est définie comme $T$ moins le triangle adjacent à $*$. Le sommet distingué \# de $T^{\prime}$ est celui de $T$.

Si le sommet $*$ participe à plusieurs triangles, on découpe la triangulation en autant de morceaux (le long des diagonales contenant $*$ ), voir la figure 2 . Le sommet $*$ donne un sommet dans chacun de ces morceaux. On prend dans chacun des morceaux le sommet à gauche de * comme sommet distingué \#. Par récurrence, on associe un chemin de Dyck à chacun des morceaux et on les concatène dans l'ordre des morceaux induit par l'ordre de gauche à droite au voisinage du sommet $*$ dans $T$, voir les figures 1 et 2 .

C'est clairement une bijection. La bijection inverse est aussi définie par récurrence sur $n$. On décompose un chemin de Dyck réductible pour la concaténation en ses composantes irréductibles et on recompose une triangulation par juxtaposition. Pour les chemins de Dyck irréductibles, on enlève une montée et une descente, on obtient une triangulation par récurrence et on rajoute un triangle.

Lemme 1.1 Le nombre de pas verticaux initiaux du chemin de Dyck $D(T)$ est le nombre de diagonales négatives dans $T$ plus 1.

Preuve. La preuve se fait par récurrence. L'énoncé est vrai pour $n=0$. On distingue deux cas comme dans la définition de la bijection. Dans le cas où $*$ est dans une seule diagonale, les deux quantités augmentent de 1. Dans l'autre cas, les deux quantités sont inchangées. 

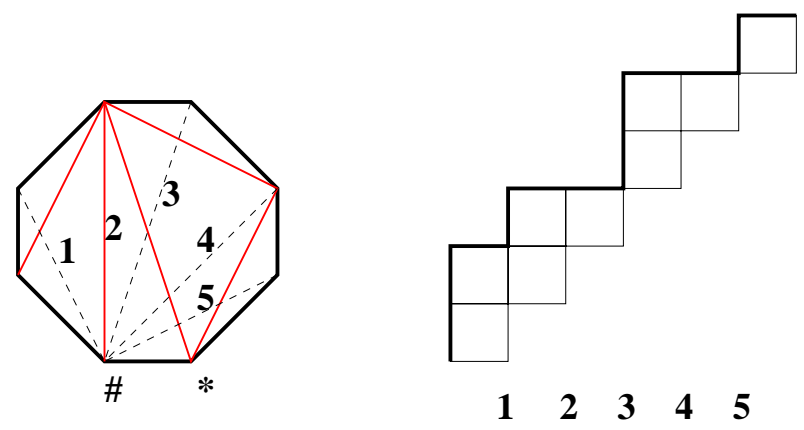

Fig. 1 - Exemple pour la bijection
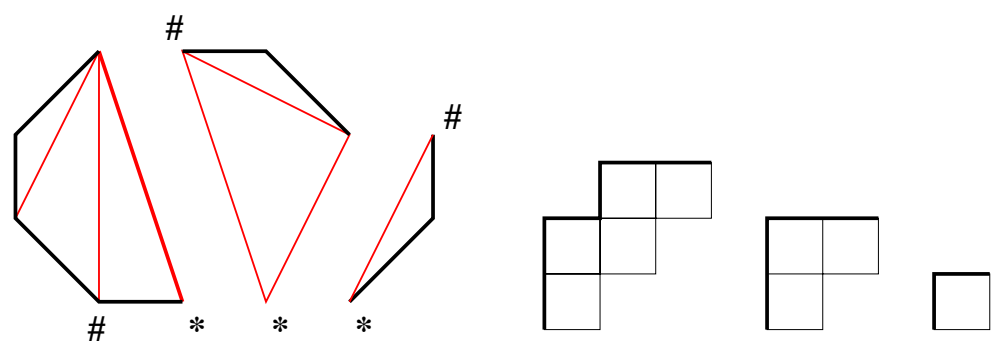

FIG. 2 - Décomposition en morceaux

\section{Polynômes}

On associe à chaque diagonale un polynôme en les variables $\left\{\mathrm{x}_{1}, \ldots, \mathrm{x}_{n+1}\right\}$ comme suit. On associe la constante 1 aux diagonales négatives. Chaque diagonale positive coupe un ensemble de diagonales négatives consécutives de $i$ à $j$. En fait, ceci donne une bijection entre les diagonales positives et les segments de $\{1, \ldots, n\}$. On peut donc parler de la diagonale positive $(i, j)$, à qui on associe alors la somme des $\mathrm{x}_{k}-\mathrm{x}_{k+1}$ pour $k=i, \ldots, j$ soit $\mathrm{x}_{i}-\mathrm{x}_{j+1}$.

On associe alors à chaque triangulation $T$ le produit $B_{T}$ des polynômes associés à ses diagonales. Dans l'exemple de la figure 1, on obtient

$$
\mathrm{B}_{T}=\left(\mathrm{x}_{1}-\mathrm{x}_{2}\right)(1)\left(\mathrm{x}_{3}-\mathrm{x}_{4}\right)\left(\mathrm{x}_{3}-\mathrm{x}_{6}\right)\left(\mathrm{x}_{5}-\mathrm{x}_{6}\right) \text {. }
$$

Par ailleurs, comme dans $[1,2]$, on associe un monôme $M_{D}$ en $\left\{\mathrm{x}_{1}, \ldots, \mathrm{x}_{n}\right\}$ à chaque chemin de Dyck $D$. On représente un chemin de Dyck par une suite de pas d'une unité vers le haut ("montée") ou vers la droite ("descente") dans une grille. On numérote les colonnes internes de la grille de 1 à $n$, voir la partie droite de la figure 1 . On convient que chaque pas vertical d'indice $i$ correspond à la variable $\mathrm{x}_{i}$. Le monôme $M_{D}$ est alors le produit des contributions des pas verticaux. Dans l'exemple de la figure 1, on obtient

$$
M_{D(T)}=\mathrm{x}_{1} \mathrm{x}_{3} \mathrm{x}_{3} \mathrm{x}_{5}
$$


On définit un ordre sur les monômes en ordonnant les variables par

$$
\mathrm{x}_{1} \ggg \mathrm{x}_{2} \ggg \cdots \mathrm{x}_{n+1} \text {. }
$$

Le monôme dominant d'un polynôme pour cet ordre est celui où intervient la plus grande puissance de $\mathrm{x}_{1}$, puis en cas d'ambiguité la plus grand puissance de $\mathrm{x}_{2}$ et ainsi de suite.

Proposition 2.1 Le monôme dominant du polynôme $\mathrm{B}_{T}$ associé à une triangulation $T$ est le monôme $M_{D(T)}$ associé au chemin de Dyck $D(T)$ correspondant à $T$ via la bijection ci-dessus.

Preuve. Par récurrence sur $n$. La proposition est vraie pour $n=0$. Soit donc $n$ non nul. On distingue deux cas.

Supposons d'abord que le sommet $*$ participe à un seul triangle de la triangulation $T$. Alors la triangulation $T$ contient la diagonale négative $n$. Le polynôme $\mathrm{B}_{T}$ ne fait donc pas intervenir $\mathrm{x}_{n+1}$ et est égal au polynôme $\mathrm{B}_{T^{\prime}}$ associé à la triangulation raccourcie en *. De même, le chemin de Dyck $D(T)$ est obtenu par concaténation d'une montée, du chemin de Dyck $D\left(T^{\prime}\right)$ et d'une descente. Donc le monôme associé à $D(T)$ est le même que celui associé au chemin $D\left(T^{\prime}\right)$. On conclut par hypothèse de récurrence que le monôme dominant de $\mathrm{B}_{T}$ est $M_{D(T)}$.

Supposons maintenant que le sommet $*$ participe à plusieurs triangles de $T$. Soit $\operatorname{Ext}(T)$ l'ensemble des nombres $k$ dans $\{1, \ldots, n\}$ tels que la diagonale négative $k$ partage un sommet avec un diagonale de $T$ contenant $*$. On va numéroter les diagonales de $T$ contenant $*$ par les éléments de $\operatorname{Ext}(T)$.

Dans la définition de $B_{T}$ comme produit sur les diagonales de $T$, on peut séparer les contributions des diagonales strictement contenues dans les différents morceaux et la contribution des diagonales de $T$ séparant les morceaux. On va traiter séparément le morceau le plus à gauche et les autres morceaux. Ces autres morceaux sont numérotés par l'élément de $\operatorname{Ext}(T)$ qui les borde sur leur gauche.

La contribution des diagonales entre les morceaux est

$$
\prod_{k \in \operatorname{Ext}(T)}\left(\mathrm{x}_{k+1}-\mathrm{x}_{n+1}\right) .
$$

Considérons le premier morceau et soit $k_{\min }$ le plus petit élément de $\operatorname{Ext}(T)$. La contribution du premier morceau est

$$
\prod_{\substack{1 \leq i \leq j<k_{\min } \\(i, j) \in T}}\left(\mathrm{x}_{i}-\mathrm{x}_{j+1}\right) .
$$

Considérons maintenant $k \in \operatorname{Ext}(T)$ et le morceau correspondant, situé à droite de $k$. Soit $k^{\prime}$ l'élément suivant de $\operatorname{Ext}(T)$ ou bien posons $k^{\prime}=n+1$ si $k$ est le plus grand élément de $\operatorname{Ext}(T)$. La contribution du morceau $k$ est alors

$$
\prod_{\substack{k+1 \leq i<k^{\prime} \\(k+1, i) \in T}}\left(\mathrm{x}_{k+1}-\mathrm{x}_{i+1}\right) \prod_{\substack{k+1<i \leq j<k^{\prime} \\(i, j) \in T}}\left(\mathrm{x}_{i}-\mathrm{x}_{j+1}\right)
$$


où le premier facteur est associé aux diagonales du morceau $k$ qui contiennent le sommet $k$.

On a donc montré que $\mathrm{B}_{T}$ est le produit de facteurs associés à chaque morceau : pour le premier morceau,

$$
\prod_{\substack{1 \leq i \leq j<k_{\min } \\(i, j) \in T}}\left(\mathrm{x}_{i}-\mathrm{x}_{j+1}\right)
$$

et, pour le morceau à droite de $k$ dans $\operatorname{Ext}(T)$,

$$
\left(\mathrm{x}_{k+1}-\mathrm{x}_{n+1}\right) \prod_{\substack{k+1 \leq i<k^{\prime} \\(k+1, i) \in T}}\left(\mathrm{x}_{k+1}-\mathrm{x}_{i+1}\right) \prod_{\substack{k+1<i \leq j<k^{\prime} \\(i, j) \in T}}\left(\mathrm{x}_{i}-\mathrm{x}_{j+1}\right)
$$

Regardons maintenant l'image $D(T)$ de $T$ par la bijection. C'est la concaténation des images des morceaux de $T$. Par définition du monôme associé, celui-ci est le produit des contributions de chaque morceau avec un décalage des indices convenable et des contributions des pas verticaux initiaux des morceaux (sauf le premier).

Par hypothèse de récurrence, la contribution du premier morceau est

$$
\prod_{\substack{1 \leq i \leq j<k_{\min } \\(i, j) \in T}} \mathrm{x}_{i}
$$

La contribution du morceau entre $k \in \operatorname{Ext}(T)$ et l'élément suivant $k^{\prime}$ de $\operatorname{Ext}(T)$ est donnée, par hypothèse de récurrence, par

$$
\mathrm{x}_{k+1}^{\ell_{k}} \prod_{\substack{k+1<i \leq j<k^{\prime} \\(i, j) \in T}} \mathrm{x}_{i}
$$

où $\ell_{k}$ est le nombre de pas verticaux initiaux du morceau $k$.

Par le lemme 1.1 appliqué au morceau $k$, on sait que le nombre $\ell_{k}$ de pas verticaux initiaux dans le morceau $k$ de $D(T)$ est égal à 1 plus le nombre de diagonales dans le morceau $k$ de $T$ qui contiennent le sommet $k$. La contribution du morceau $k$ au monôme $M_{D(T)}$ est donc

$$
\mathrm{x}_{k+1} \prod_{\substack{k+1 \leq i<k^{\prime} \\(k+1, i) \in T}} \mathrm{x}_{k+1} \prod_{\substack{k+1<i \leq j<k^{\prime} \\(i, j) \in T}} \mathrm{x}_{i}
$$

On vérifie que le terme dominant de la contribution de chaque morceau à $\mathrm{B}_{T}$ est bien égal à la contribution de chaque morceau à $M_{D(T)}$. En prenant le produit des contributions des morceaux, on obtient l'égalité voulue.

Théorème 2.2 Les polynômes $\mathrm{B}_{T}$ associés aux triangulations forment une base de l'algèbre des coinvariants quasi-symétriques. Cette base est stable par le renversement des variables $\mathrm{x}_{i} \mapsto \mathrm{x}_{n+2-i}$. Les deux choix naturels d'ordre total sur les variables donnent deux bases monomiales, en prenant les monômes dominants des polynômes $\mathrm{B}_{T}$. 
Preuve. Dans [1], il est démontré que les classes des monômes $M_{D}$ associés aux chemins de Dyck forment une base de l'anneau des coinvariants quasi-symétriques. On déduit alors de la proposition 2.1 que les classes des polynômes $\mathrm{B}_{T}$ forment aussi une base. Le fait que cette base soit stable par le renversement est immédiat : l'image de $\mathrm{B}_{T}$ est $\mathrm{B}_{T^{\prime}}$ où la triangulation $T^{\prime}$ est obtenue par renversement de $T$. Enfin la dernière assertion est juste une reformulation de la proposition 2.1 et son image par le renversement.

\section{Références}

[1] J.-C. Aval, F. Bergeron, and N. Bergeron. Ideals of quasi-symmetric functions and super-covariant polynomials for $S_{n}$. Adv. Math., 181(2) :353-367, 2004.

[2] J.-C. Aval and N. Bergeron. Catalan paths and quasi-symmetric functions. Proc. Amer. Math. Soc., 131(4) :1053-1062 (electronic), 2003.

[3] P. Caldero, F. Chapoton, and R. Schiffler. Quivers with relations arising from clusters ( $A_{n}$ case). T.A.M.S., 2005.

[4] S. Fomin and A. Zelevinsky. Cluster algebras. II. Finite type classification. Invent. Math., 154(1) :63-121, 2003.

[5] Ira M. Gessel. Multipartite $P$-partitions and inner products of skew Schur functions. In Combinatorics and algebra (Boulder, Colo., 1983), volume 34 of Contemp. Math., pages 289-317. Amer. Math. Soc., Providence, RI, 1984.

[6] R. Steinberg. Differential equations invariant under finite reflection groups. Trans. Amer. Math. Soc., 112 :392-400, 1964. 